\section{Tunable single- and dual-wavelength fiber ring lasers using an Er-Yb doped waveguide amplifier}

\author{
Peng-Chun Peng \\ National Chiao-Tung University \\ Institute of Electro-Optical Engineering \\ Hsinchu, Taiwan 300
}

\section{Sien Chi}

National Chiao-Tung University

Institute of Electro-Optical Engineering

Hsinchu, Taiwan 300

and

Yuan Ze University

Department of Electrical Engineering

Chungli, Taiwan 320

\begin{abstract}
We demonstrate tunable single- and dualwavelength fiber ring lasers using a compact Er-Yb doped waveguide amplifier. In the single-wavelength operation, a signal-to-noise ratio higher than $63 \mathrm{~dB}$ and an output power maintaining around $11 \mathrm{dBm}$ in the operation range over $50 \mathrm{~nm}$ (from 1529.1 to $1579.43 \mathrm{~nm}$ ) are achieved. In the dual-wavelength operation, lasing wavelengths with wavelength separation from 51.73 to $6.2 \mathrm{~nm}$ are demonstrated. (C) 2005 Society of Photo-Optical Instrumentation Engineers. [DOI: 10.1117/1.1927487]
\end{abstract}

Subject terms: fiber ring laser; dual-wavelength laser; tunable laser; $\mathrm{Er}-\mathrm{Yb}$ doped waveguide amplifier.

Paper L040316R received May 26, 2004; revised manuscript received Mar. 10, 2005; accepted for publication Apr. 13, 2005; appeared online Apr. 18, 2005; published online Jun. 20, 2005.

\section{Introduction}

Great efforts have been made on the development of the Er-Yb doped waveguide amplifier (EYDWA) using ion exchange technology in planar glass integrated optics. ${ }^{1}$ This device might become a key component in wavelengthdivision-multiplexing (WDM) systems because of its compactness and excellent compatibility with optical fibers. ${ }^{2-4}$ Recently, it has been proposed to combine the EYDWA and fiber Bragg gratings to realize single- and multi-wavelength laser sources. ${ }^{5,6}$

Tunable fiber lasers are applicable to loss measurements of optical components, optical sensing systems, ${ }^{7}$ and tunable transmitters in WDM systems. In this paper, we experimentally investigate and demonstrate tunable singleand dual-wavelength fiber ring lasers using a compact EYDWA. These fiber lasers are of small size, have a wide tunable range, and deliver high output power. The performances of tunable range, output power, and signal-to-noise ratio (SNR) are studied.

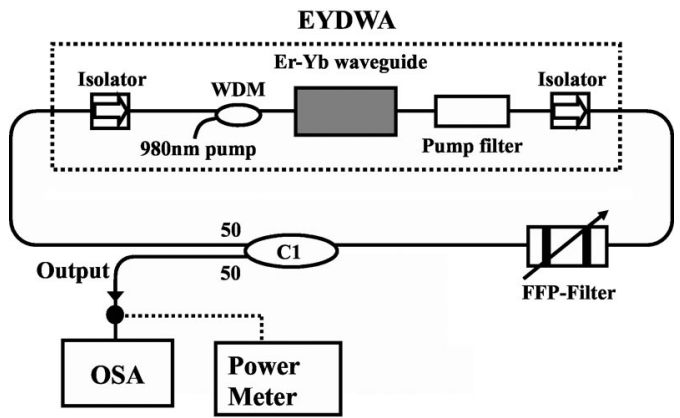

E

L

E

Fig. 1 Experimental setup of the tunable single-wavelength fiber laser. (C1: $1 \times 2$ coupler with coupling ratio 50:50, WDM: 980/ 1550-nm WDM coupler, FFP-filter: fiber Fabry-Perot filter, OSA: optical spectrum analyzer).

\section{Experiments and Results}

Figure 1 shows the experimental setup for the tunable single-wavelength fiber ring laser using a EYDWA. This configuration is constructed by a $1 \times 2$ optical coupler with coupling ratio 50:50 (C1), a fiber Fabry-Perot filter (FFP filter), and a EYDWA. The EYDWA with a saturated output power $16.5 \mathrm{dBm}$ is manufactured by Teem Photonics via a two-step ion exchange process. The FFP filter is an all-fiber device with a wide tunable range, low insertion loss, and low polarization dependence. For these reasons, we choose the FFP filter for the intracavity filter. The central wavelength of the FFP filter is tuned by the voltage applied to the piezoelectric transducer. The free spectral range (FSR) and 3-dB bandwidth of the FFP filter are $51.73 \mathrm{~nm}$ and 0.53 $\mathrm{nm}$. The insertion loss of the FFP filter is $1.9 \mathrm{~dB}$. An optical spectrum analyzer (OSA) and a power meter are used to measure the output spectra and powers of the fiber laser. Figure 2 shows the optical spectra of the tunable singlewavelength fiber laser while the various voltages are applied on the FFP filter. The lasing wavelength tuning over $50 \mathrm{~nm}$ (from 1529.1 to $1579.43 \mathrm{~nm}$ ) is observed. The output power is maintained around $11 \mathrm{dBm}$, and the SNR can be kept higher than $63 \mathrm{~dB}$ in a wide tuning range. The cavity length and longitudinal mode spacing are about $24 \mathrm{~m}$ and $8.3 \mathrm{MHz}$, respectively.

Because the FSR of the FFP filter is $51.73 \mathrm{~nm}$, the dualwavelength lasing is observed when the FFP filter is tuned to $1580.48 \mathrm{~nm}$, as shown in Fig. 3. This result shows the

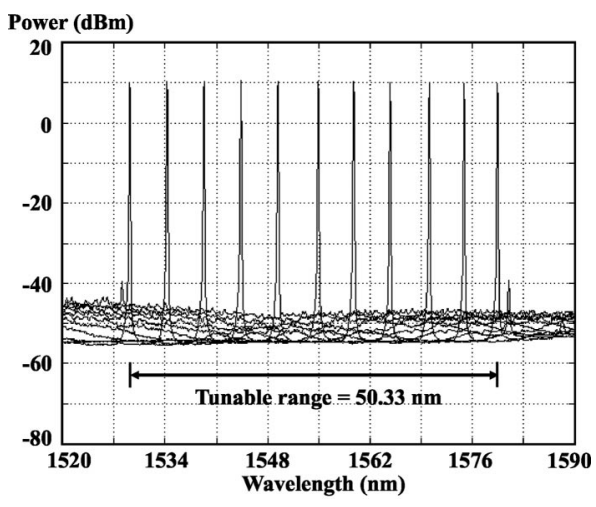

Fig. 2 Output spectra of the tunable single-wavelength fiber laser with various voltages applied on the FFP filter. 


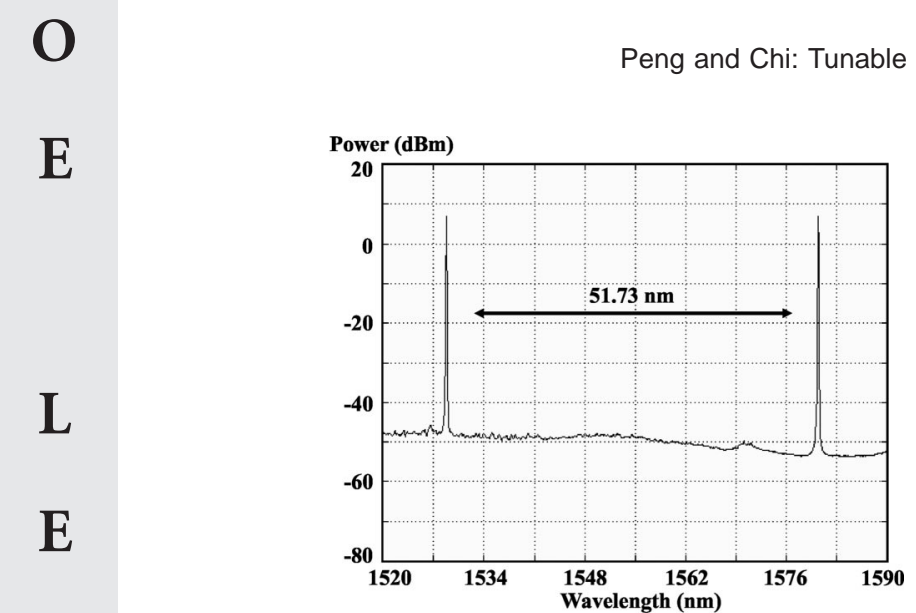

$\mathrm{T}$

$\mathrm{T}$

E

$\mathbf{R}$ wavelength.
Fig. 3 Output spectrum of the fiber laser when the central wavelength of the FFP filter is tuned to $1580.48 \mathrm{~nm}$.

potentially tunable dual- or multi-wavelength lasing in the whole range of 1528.75 to $1580.48 \mathrm{~nm}$. However, one of the major problems in the tunable dual- or multiwavelength fiber laser using a EYDWA at room temperature is correctly adjusting the cavity losses on each lasing

We set up an experiment for the tunable dualwavelength fiber laser using a EYDWA, as shown in Fig. 4. In our experiment, the tunable dual-wavelength fiber laser consists of a EYDWA, two FFP filters, two variable attenuators (VA), a $2 \times 2$ optical coupler (C2), and a $1 \times 2$ optical coupler (C3). Due to the homogeneous gain broadening of the EYDWA, individual loss control for different wavelengths is required. To solve this problem, the variable attenuators are used to adjust the losses at different lasing wavelengths. Furthermore, the variable attenuators can be adjusted to equalize the power level of the lasing wavelengths. Figures 5(a)-(d) shows the output spectra of the tunable dual-wavelength laser when the FFP filters are tuned at different wavelengths. The wavelength separations of the two lasing wavelengths are $42.5,30,18$, and $6.2 \mathrm{~nm}$, and the differences in loss between the two cavities are 1.6, $3.2,3.7$, and $2.1 \mathrm{~dB}$, respectively. Moreover, we continuously monitor the two wavelengths for $10 \mathrm{~min}$. Power variation of both lasing wavelengths is less than $1.5 \mathrm{~dB}$.

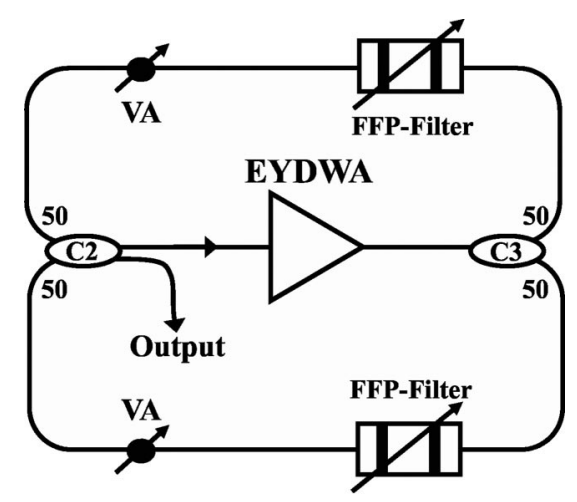

Fig. 4 Experimental setup of the tunable dual-wavelength fiber laser. (C2: $2 \times 2$ coupler with coupling ratio 50:50, C3: $1 \times 2$ coupler with coupling ratio 50:50, VA: variable attenuator).

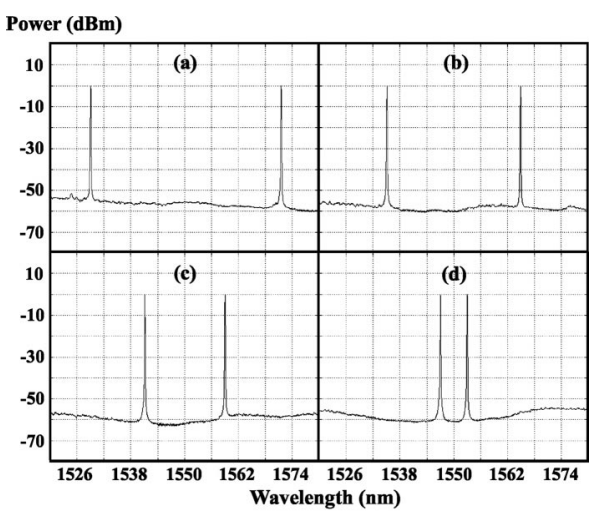

Fig. 5 Output spectra of the dual-wavelength fiber laser with various wavelength separations: (a) $42.5 \mathrm{~nm}$, (b) $30 \mathrm{~nm}$, (c) $18 \mathrm{~nm}$, and (d) $6.2 \mathrm{~nm}$.

These experiments show potentially multi-wavelength lasing in the whole range of 1528.75 to $1580.48 \mathrm{~nm}$.

\section{Conclusion}

In summary, tunable single- and dual-wavelength fiber ring lasers based on a compact EYDWA have been experimentally investigated and demonstrated. In the singlewavelength operation, a SNR higher than $63 \mathrm{~dB}$ and an output power maintaining around $11 \mathrm{dBm}$ in the operation range over $50 \mathrm{~nm}$ (from 1529.1 to $1579.43 \mathrm{~nm}$ ) have been achieved. In the dual-wavelength operation, the lasing wavelengths with wavelength separation from 51.73 to 6.2 $\mathrm{nm}$ are demonstrated. These results show that tunable lasers using EYDWA are potentially acceptable for WDM applications.

\section{Acknowledgment}

This work was supported in part by the MediaTek Fellowship and in part by the National Science Council of the Republic of China under Contract NSC 93-2752-E-009009-PAE and Contract NSC 93-2215-E-155-005.

\section{References}

1. D. Barbier, J. M. P. Delavaux, and A. Kevorkian, "Yb/Er integrated optics amplifiers on phosphate glass in single and double pass configuration," Optical Fiber Communication Conference '95, San Diego, CA, postdeadline paper PD3-1 (1995).

2. D. R. Zimmerman and L. H. Spiekman, "Amplifiers for the masses: EDFA, EDWA, and SOA amplets for metro and access applications," J. Lightwave Technol. 22(1), 63-70 (2004).

3. S. Blaize, L. Bastard, C. Cassagnetes, and J. E. Broquin, "Multiwavelengths DFB waveguide laser arrays in $\mathrm{Yb}$-Er codoped phosphate glass substrate," IEEE Photonics Technol. Lett. 15(4), 516-518 (2003).

4. K. C. Reichmann, P. P. Iannone, M. Birk, N. J. Frigo, D. Barbier, C. Cassagnettes, T. Garret, A. Verlucco, S. Perrier, and J. Philipsen, "An eight-wavelength 160-km transparent metro WDM ring network featuring cascaded erbium-doped waveguide amplifiers," IEEE Photonics Technol. Lett. 13(10), 1130-1132 (2001).

5. G. Sorbello, S. Taccheo, P. Laporta, O. Svelto, E. Cianci, V. Foglietti, S. Jiang, and N. Peyghambarian, "Singlemode Er:Yb waveguide laser array at $1.5 \mu \mathrm{m}, "$ Electron. Lett. 37(16), 1014-1015 (2001).

6. G. Jose, S. Taccheo, G. Sorbello, D. Migliorati, V. Foglietti, E. Cianci, S. Jiang, N. Peyghambarian, and P. Laporta, "Multiwavelength waveguide laser array in C-band," Electron. Lett. 38(21), 1275-1276 (2002).

7. P. C. Peng, H. Y. Tseng, and S. Chi, "A novel fiber-laser-based sensor network with self-healing function," IEEE Photonics Technol. Lett. 15(2), 275-277 (2003). 